\title{
Consumo de alcohol y riesgo de accidente cerebrovascular
}

Alcohol Consumption and Risk of Stroke. Reynolds K, Lewis BL, Nolen JD. JAMA 2003,289(5):579-588

\section{Objetivo}

Examinar la asociación entre consumo de alcohol y el riesgo de Accidente cerebrovascular (ACV).

\section{Fuente, Selección y extracción de Datos}

Búsqueda en MEDLINE desde 1996 a 2002 y de referencias principales limitado a literatura de habla inglesa que identificó 122 estudios. Se incluyeron 35 estudios observacionales, 19 de cohorte y 16 caso-control, en los cuales el resultado fue ACV, isquémicos, hemorrágicos o totales. Se evaluaron variables demográficas, tabaquismo, hipertensión arterial (HTA), enfermedad cardiovascular, hormonoterapia de reemplazo y determinaciones de colesterol. Tres investigadores independientes extrajeron los datos siguiendo un protocolo.

\section{Resultados principales}

Los estudio de cohortes totalizaron 486.591 pacientes, siete incluyeron hombres y mujeres, 11 sólo hombres y 1 sólo mujeres. EI rango de edad fue entre 18 y 90 años y el de seguimiento, entre 4 y 30 años.

En los estudios caso-control, hubo 4.469 casos y 6329 controles de ambos sexos.

Se establecieron cuatro categorías de consumo diario ( $<12 \mathrm{~g} /$ día, 12 a $24 \mathrm{~g} /$ día, 24 a 60g/día y >60g/día) comparándolas con los no consumidores.

Comparado con los abstemios, el consumo $>60 \mathrm{~g} /$ día se asoció con un aumento del riesgo de ACV (RR 1,64 de ACV totales, 1,69 de ACV isquémico y 2,1 ACV hemorrágico) mientras el consumo $<12 \mathrm{~g} /$ día redujo el RR de ACV totales a 0.83 y de $\mathrm{ACV}$ isquémico a 0,80; y el consumo de 12 a 14g/día redujo el RR a 0,72 (ver cuadro 1). El análisis de regresión reveló asociación no lineal entre el consumo de alcohol y el de ACV totales e isquémicos y asociación lineal con consumo de alcohol y ACV hemorrágico.

La asociación fue similar en hombres y mujeres con un RR algo menor en las mujeres consumidoras $<12 \mathrm{~g} /$ día respecto a los hombres del mismo subgrupo. Asimismo, la asociación fue similar en los estudios de cohorte y en los de caso-control.

Cuadro 1: riesgo relativo (IC95\%) de ACV asociado al consumo de alcohol.

\begin{tabular}{l|l|l|l|l|l} 
& NNo de & \multicolumn{4}{|c}{ Consumo de alcohol } \\
\cline { 3 - 7 } & Estudios & $<12$ g/dia & $\mathbf{1 2}$ a 24g/dia & $\mathbf{2 4}$ a 60g/dia & $>$ 60g/día \\
\hline ACV totales & 35 & $0,83(0,75-0,91)$ & $0,91(0,78-1,06)$ & $1,10(0,97-1,24)$ & $1,64(1,39-1,93)$ \\
\hline Isquémicos & 15 & $0,80(0,67-0,96)$ & $0,72(0,57-0,91)$ & $0,96(0,79-1,18)$ & $1,69(1,34-2,15)$ \\
\hline Hemorrágicos & 12 & $0,79(0,60-1,05)$ & $0,98(0,77-1,25)$ & $1,19(0,80-1,79)$ & $2,18(1,48-3,20)$ \\
\hline En hombres & 27 & $0,89(0,79-1,01)$ & $0,94(0,84-1,05)$ & $1,08(0,96-1,21)$ & $1,76(1,57-1,98)$ \\
\hline En mujeres & 16 & $0,66(0,61-0,71)$ & $0,79(0,56-1,11)$ & $0,80(0,49-1,30)$ & $4,29(1,3-14,14)$ \\
\hline Cohortes & 19 & $0,82(0,83-0,92)$ & $0,94(0,84-1,05)$ & $1,06(0,90-1,23)$ & $1,63(1,49-1,79)$ \\
\hline Casos control & 16 & $0,80(0,67-0,97)$ & $0,65(0,44-0,96)$ & $1,12(0,92-1,37)$ & $1,98(1,35-2,92)$ \\
\hline
\end{tabular}

\section{Conclusiones}

El consumo pesado de alcohol aumenta el riesgo de ACV mientras que el consumo ligero a moderado disminuiría los ACV, fundamentalmente a expensas de los isquémicos.

Fuente de financiamiento: National Heart, Lung and Blood Institute.

\section{Comentario}

El consumo de alcohol está muy difundido en todo el mundo y su asociación con el ACV podría tener implicancias clínicas individuales y sobre la Salud Pública dado que es una de las primeras causas de muerte e incapacidad permanente y con gran repercusión en el presupuesto sanitario $0^{1,2}$. En la relación entre el consumo de alcohol y ACV están involucrados mecanismos como la hipertensión inducida por alcohol, las cardiomiopatías, los desórdenes de la coagulación y la reducción del flujo cerebral. El efecto antiagregante plaquetario del alcohol, el efecto hipertensivo y los desórdenes de la coagulación explicarían el incremento del riesgo de ACV hemorrágico; mientras que el aumento del colesterol HDL, la disminución del ACV isquémico.

Muchos estudios epidemiológicos han examinado el rol del alcohol ${ }^{3,4}$ tanto como factor de riesgo ${ }^{5,6}$ como de protección del ACV7, por lo que la realización de este meta-análisis estaba plenamente justificada.

La limitación de éste estudio es la calidad de los datos de los es- tudios observacionales incluidos. Por ejemplo, algunos no contaban con diagnóstico tomográfico y se usaron diferentes medidas de consumo de alcohol. Sin embargo las conclusiones son extrapolables a otros grupos de pacientes, ya que incluyó un elevado número de personas de distintas partes del mundo, se validaron las medidas de consumo de alcohol, la consistencia de los hallazgos en el análisis de subgrupos y se efectuó análisis de regresión múltiple sin variación de los resultados luego de corregir por los potenciales efectos confundidores.

Conclusiones del comentador: Teniendo en cuenta la difusión del consumo de alcohol en la población general, sería interesante la difusión de medidas tendientes a la prevención del ACV como la reducción del consumo pesado de alcohol. Si bien el consumo moderado de alcohol reduciría el riesgo de ACV isquémico, debería valorarse en cada paciente individual la relación riesgo/beneficio.

Dra. Haydée Giraudi [ Servicio de Salud Ocupacional. Hospital Italiano de Buenos Aires ]

\footnotetext{
Referencias

1. American Heart Association. 2002 Heart and Stroke Statistical Update. Dallas, Tex: American Heart Association; 2001.

2. Warlow CP. Epidemiology of stroke. Lancet. 1998;352(suppl 3):1-4.
3. Donahue RP, Abbott RD, Reed DM, Yano K. Alcohol and hemorrhagic stroke: the Honolulu Heart Program. JAMA. 1986;255:2311-2314.

4. Malarcher AM, Giles WH, Croft JB, et al. Alcohol intake, type of beverage, and the risk of cerebral infarction in young women. Stroke. 2001;32:77-83.

5. Gill JS, Zezulka AV, Shipley MJ, Gill SK, Beevers DG. Stroke and alcohol consumption. N Engl J Med. 1986;315:1041-1046.

6. Palomäki H, Kaste M. Regular light-to-moderate intake of alcohol and the risk of ischemic stroke: is there a beneficial effect? Stroke. 1993;24:1828-1832.

7. Jamrozik K, Broadhurst RJ, Anderson CS, Stewart-Wynne EG. The role of lifestyle factors in the etiology of stroke: a population-based case-control study in Perth, Western Australia. Stroke. 1994;25:5159 .
} 